\title{
A PESQUISA NO CAMPO DE PROJETO DE ARQUITETURA E URBANISMO NO BRASLL NA PERSPECTIVA DOS SEMINÁRIOS PROJETAR 2003-2013
}

RESEARCH IN THE FIELD OF ARCHITECTURAL AND URBAN DESIGN IN BRAZIL From THE PERSPECTIVE OF PROJETAR SEMINARS 2003-2013

LA INVESTIGACIÓN EN EL CAMPO DEL PROYECTO ARQUITECTÓNICO Y URBANO EN BRASIL DESDE LA PERSPECTIVA DE LOS SEMINARIOS PROJETAR २०03-2013

\section{VELOSO, MAÍSA}

Doutora, Professora Associada da UFRN, maisaveloso@gmail.com

\begin{abstract}
RESUMO
Neste texto, discute-se a evolução das investigações científicas que se dizem vinculadas à linha de pesquisa em projeto de arquitetura e/ou urbanismo no Brasil nos últimos 12 anos, a partir da produção intelectual apresentada desde a primeira edição do Seminário PROJETAR em Natal/2003, evento que tem como foco principal de discussão o ensino, a pesquisa e a prática profissional neste campo. Buscando compreender o que e como se produz cientificamente neste campo, questionam-se: Quais os recortes temáticos e os principais objetos de análise? Que suportes teóricos e metodológicos fundamentam essas pesquisas? São mesmo pesquisas sobre o projeto ou que visam Ihe dar suporte? Mas o que é uma pesquisa em projeto? E como se distingue das outras áreas de conhecimento da Arquitetura? Essa discussão terá como bases principais a revisão da restrita literatura sobre este tema e a análise dos artigos constantes dos anais das seis edições anteriores dos Seminários PROJETAR (2003, 2005, 2007, 2009, 2011, 2013). As análises dos dados levantados permitiram concluir que ainda persistem divergências conceituais e metodológicas importantes no que se entende como pesquisa neste campo específico do conhecimento, assim como a hegemonia das discussões sobre o ensino do projeto em nível de graduação (formação profissional) no âmbito dos Seminários PROJETAR.
\end{abstract}

PALAVRAS-CHAVE: arquitetura, urbanismo, projeto, pesquisa científica.

\section{ABSTRACT}

In this paper, we discuss the evolution of scientific investigations linked to the line of research in architectural and / or urban design in Brazil over the past 12 years, based on the intellectual production presented since the first edition of PROJETAR Seminar in Natal / 2003, event whose main focus of discussion are teaching, research and professional practice in this field. Trying to understand what and how has been produced scientifically in this field, the questions are: What are the thematic cutouts and the main analysis objects? What theoretical and methodological supports underlie these surveys? They are even research on the project or seek to support it? But what is a research in project? And how it is distinguished from other areas of knowledge of architecture? This discussion will have as main bases reviewing the limited literature on this topic and the analysis of the articles of the annals of the six previous editions of the PROJETAR Seminars (2003, 2005, 2007, 2009, 2011 and 2013). Analyses of data collected showed that there are still important conceptual and methodological differences in what is understood as research in this specific field of knowledge, as well as the hegemony of the discussions on the teaching of design at the undergraduate level in PROJETAR Seminars.

KEY-WORDS: architecture, urbanism, design, scientific research.

\section{RESUMEN}

En este trabajo, se discute la evolución de las investigaciones científicas vinculados a la línea de investigación en el proyecto arquitectónico y urbano en Brasil en los últimos 12 años, a partir de la producción intelectual que se presenta desde la primera edición del Seminario PROJETAR / 2003 en Natal, evento que se centra principalmente en la enseñanza, la investigación y la práctica profesional en este campo. Tratar de entender qué y cómo producir científicamente en este campo, la pregunta es: ¿Cuáles son los recortes temáticos y los principales objetos de análisis? ¿Qué apoyos teóricos y metodológicos subyacen estas encuestas? Son incluso la investigación sobre el proyecto o buscan apoyo para este? Pero lo que es una investigación en proyecto? ¿Y cómo se distingue de otras áreas del conocimiento de la arquitectura? Esta discusión tendrá como principales bases la revisión de la literatura limitada sobre este tema y el análisis de los artículos de los anales de las seis ediciones anteriores de los Seminarios PROJETAR (2003, 2005, 2007, 2009, 2011, 2013). Los análisis de los datos recogidos muestran que todavía hay diferencias conceptuales y metodológicas importantes en lo que se entiende como la investigación en este campo específico del conocimiento, así como la hegemonía de los debates sobre la enseñanza del proyecto a nivel de pregrado (formación profesional) en los Seminarios PROJETAR.

PALABRAS-CLAVE: arquitectura, urbanismo, proyecto, investigación científica. 


\section{INTRODUÇÃO}

O Seminário PROJETAR apresenta em sua proposta inicial (primeira edição, Natal/2003) a discussão sobre o ensino e a pesquisa no campo do projeto de arquitetura. Ao longo de suas edições subsequentes foram-se paulatinamente incorporando a intervenção projetual na escala urbana e a dimensão prática da profissão e da atuação acadêmica através de projetos de extensão em Arquitetura e Urbanismo (AU). Conciliando as atividades de pesquisa desenvolvidas no âmbito do Grupo Projetar da UFRN (fundador do Seminário) e a organização desta sétima edição do evento (2015), que retorna a Natal e se propõe a fazer uma avaliação de seus 12 anos de trajetória, fizemos uma leitura crítica dos anais das seis edições anteriores do Seminário (2003, 2005, 2007, 2009, 2011, 2013). Essa leitura revelou alguns dados de interesse para o delineamento de um panorama da pesquisa em/sobre o projeto de AU no Brasil nos últimos anos. Considera-se que a produção intelectual dos Seminários PROJETAR é um importante indicador da investigação científica neste campo no Brasil, envolvendo, sobretudo, neste recorte, a pesquisa de pós-graduação. Esta leitura foi aprofundada com o olhar dirigido para dois conjuntos de questões: i) $\bigcirc$ que se entende por pesquisa no campo do projeto e como se distingue das outras subáreas de conhecimento da Arquitetura e do Urbanismo? ii) $\mathrm{O}$ que se tem apresentado como os produtos de pesquisas inseridas neste domínio no âmbito dos Seminários PROJETAR 2003-2013? Quais os recortes temáticos e os principais objetos de análise? Que suportes teóricos e metodológicos fundamentam essas pesquisas? São mesmo pesquisas sobre o projeto ou que visam Ihe dar suporte?

Além deste rico material primário (os artigos publicados nos anais de seis edições do evento que se apresentaram como produtos de pesquisas), as análises aqui apresentadas foram também fundamentadas em documentos e bibliografia de referência para esta discussão, como documentos dos órgãos de fomento à pesquisa e à pós-graduação no Brasil e, secundariamente, em discussões correlatas sobre o tema da investigação neste campo realizadas em outros fóruns que não o PROJETAR.

Deve-se destacar que a quarta edição do PROJETAR (São Paulo, 2009) teve a temática central dirigida para o projeto como investigação, o que representou importante avanço para a discussão. Comparando com o panorama traçado nos meados da década passada, podemos identificar quais os avanços e os limites das investigações neste domínio do conhecimento e o que o surgiu de novo desde 2003. Espera-se também dialogar em mesa redonda com outros pesquisadores/professores com experiência de pesquisa e ensino na linha de projeto, delineando pontos comuns, lacunas e possibilidades de pesquisas futuras.

\section{A PESQUISA NO CAMPO DO PROJETO DE ARQUITETURA E URBANISMO NO BRASIL}

A pesquisa na área de projeto é relativamente recente no Brasil. Por muito tempo associado à prática profissional, o projeto quase sempre assumiu papel central na formação em nível de graduação nos cursos de AU no país, mas dificilmente eram encontrados laboratórios ou grupos de pesquisa neste campo. Com o crescimento das pós-graduações a partir dos anos 1990, e a necessidade de qualificação pós-graduada para os docentes (inclusive os de projeto), esse quadro começa a mudar. Em 2003, Veloso e Elali fizeram um levantamento da estrutura curricular dos programas de pós- graduação (PPG) em AU existentes à época e identificaram que 6 (ou 42\%) dos 14 PPG apresentavam área de concentração em projeto de arquitetura, projeto de arquitetura e urbanismo ou projeto do ambiente construído, sendo que apenas $02 \mathrm{em}$ projeto de arquitetura isoladamente (VELOSO e ELALI, 2003). Passados 12 anos, hoje são 36 PPG em AU e a incidência de áreas de concentração com foco no projeto é apenas um pouco superior, correspondendo a com 44,5\% dos programas recomendados pela CAPES. Deve-se destacar uma maior presença deste foco nos programas mais novos e nos recém-criados mestrados profissionais.

Em 2003, o Grupo de Pesquisa e o Seminário PROJETAR surgiram juntos na UFRN visando impulsionar estudos e pesquisas científicas neste campo, em sintonia com as tendências internacionais à época. Além deste evento pioneiro no Brasil, outros encontros como os ocorridos em

Marselha (2004), Princeton (2004), Dublin (2004), Edimburgo (2006) - parecem atestar uma tendência nacional e internacional - pelo menos ocidental - da pesquisa da arquitetura, tendo como objeto central de investigação o projeto, e, deste modo, inaugurar um novo momento da cultura arquitetural (VELOSO e MARQUES, 2007, sp). 
As edições subsequentes dos Seminários PROJETAR, e notadamente a de 2009 em São Paulo, dão destaque para o projeto como um campo profícuo de investigação, inclusive obtendo recursos para financiamento de pesquisas e fóruns de debate, o que era muito difícil em décadas passadas. Esses avanços levaram ao reconhecimento da pesquisa científica na área de projeto o que passa a ser inclusive expresso em documentos oficiais dos órgãos de fomento no Brasil, como, por exemplo, a CAPES.

Segundo o Documento da área de Arquitetura e Urbanismo e Design junto a esse órgão governamental, os princípios gerais que norteiam o ensino e a pesquisa em pós-graduação na área são, dentre outros: 1. Interdisciplinaridade e transversalidade na abordagem dos temas tratados, buscando superar os limites da visão disciplinar e setorial; 2. Articulação entre teoria e prática para a compreensão da realidade contemporânea; 3. Apropriação das dimensões tempo e espaço e suas diferentes escalas; e 4. Entendimento do projeto como elemento articulador de pesquisas, e seus desdobramentos nos estudos teórico-críticos (...) (CAPES, 2013, AU e D, pp.01-02, grifos nossos).

Ainda segundo o mesmo documento, uma discussão frequente na área, trata de sua especificidade e de suas ações. Nos programas de pós-graduação,

projeto, tecnologia e história figuram de maneira não homogênea na declaração de campo de interesse, com especial prevalência do campo de história na identificação de áreas de concentração ou linhas de pesquisa. Assumindo como importante uma interlocução mais estreita com as demandas da sociedade contemporânea, projeto (tema fundamental nas duas subáreas) e tecnologia devem, naturalmente, passar a figurar com mais frequência nas ações de investigação promovidas pelos programas, uma vez que as novas demandas direcionarão os investimentos (de tempo e de recursos) no processo de qualificação acadêmica e profissional. Como resultado, parcerias com outras Áreas podem vir a apontar novas alternativas nesses campos de atuação, dando início a um ciclo virtuoso de renovação do conhecimento e das alternativas de sua aplicação. (CAPES, $A \cup$ e D, 2013, pp.03-04).

O projeto passa enfim a ser oficialmente reconhecido como campo profícuo de pesquisa de pós-graduação e que deve ser inclusive incentivado. Mas o que é a pesquisa em projeto? Pesquisa-se sobre o projeto, para o projeto, com base em ou através de projetos?
Para Malard, deve-se assumir que existe uma dificuldade real em se diferenciar o que seja uma pesquisa para se fazer um projeto e o que seja uma pesquisa para se desenvolver conhecimento na área de projeto, pois em ambos os casos, pode ocorrer a produção de conhecimento. "A nossa visão é de que qualquer projeto de arquitetura implica necessariamente num processo de pesquisa, independentemente de sua complexidade conceitual, funcional, tecnológica ou plástica" (MALARD, sd). Evidentemente essa visão está mais relacionada ao fazer projetual. Ao pesquisar para projetar, entendendo essa pesquisa como passível de gerar conhecimentos em Arquitetura e Urbanismo. Em ambos os casos, parte-se de um problema que o projeto pretende resolver por meio de análise, avaliação e síntese/proposição.

Outra visão possível é o estudo do projeto (e não obra construída) como documento e registro da produção arquitetônica e urbanística de uma época, constituindo fonte de investigação e de produção de novos conhecimentos (Boutinet, 2000; Chupin, 2004; Lawson, 2011).

No encontro em Marselha em 2004 (EURAU'04), a questão dos emergentes doutorados de arquitetura foi discutida e dentre outros temas tratados a relação entre pesquisa científica e projeto. Superadas as incertezas iniciais quanto aos possíveis objetos de uma tese na área de projeto, reconhece-se que, se o ato de projetar em si não é necessariamente científico, os processos e produtos nele envolvidos podem sim ser objeto de uma reflexão teórica-conceitual e metodológica com procedimentos próprios de uma metodologia científica. A questão do que seria um corpus teórico-conceitual e metodológico próprio ao saber projetual permanece em aberto, uma vez que se reconhece a natureza aberta e relativamente mal definida deste campo do conhecimento, inserido no que Herbert Simon, desde os anos 1960, cunhou de "ciências do artificial", isto é, aquelas que envolvem a criação de artefatos por meio da inventividade humana.

Internacionalmente, devemos destacar os trabalhos da Design Research Society (fundada como desdobramento da Design Methods Conference na década de 60), e as abordagens do Project Based Research e Pratice Based Research que procuram articular pesquisa acadêmica e práticas projetuais. É notável também 
os trabalhos desenvolvidos pelo Leap (Laboratoire d'étude de l'architecture potentielle) da Universidade de Montreal, principalmente sobre o rico material gerado pelos projetos participantes de concursos de arquitetura, e do recém-criado laboratório denominado Project[s] da ENSA-Marselha (1)

Como exemplo de pesquisas na área de projeto no Brasil, poderíamos aqui citar, dentre outras, aquelas desenvolvidas nos últimos anos no âmbito de grupos como ProLUGAR da UFRJ, Arquitetura: Projeto \& Pesquisa \& Ensino da Universidade Presbiteriana Mackenzie de São Paulo, assim como as do Grupo Projetar/UFRN (2). As investigações deste último grupo abordam o projeto como fonte de conhecimento por meio de estudos sobre teoria da arquitetura e do projeto, métodos e técnicas de projetação, de avaliação projetual e de ensino/ aprendizagem na área de projeto, envolvendo questões teóricas e metodológicas mais amplas, embora com trabalhos aplicados a recortes temáticos específicos, como a arquitetura residencial, escolar, hospitalar, processos de projetos e obras de arquitetos renomados, e a conservação, restauro e requalificação do patrimônio edificado.

Uma outra linha de pesquisa procura entender em que medida a prática projetual em arquitetura e urbanismo produz ou vem produzindo conhecimentos que possam ser validados no âmbito acadêmico ou mesmo constituindo um "método projetual de investigação e pesquisa", baseado sobretudo em artefatos não textuais. A pesquisa do Grupo da UPM (Lima et al, 2011) examinou sistematicamente um vasto universo de trabalhos acadêmicos (mestrados e teses de doutoramento) realizados na FAUUSP, instituição tradicional na formação pós-graduada no Brasil e constatou haver

uma importante frequência de elementos característicos da prática projetual que comparecem, em maior ou menor grau conforme cada caso, como parte essencial da argumentação desses trabalhos. Mas ainda não foi possível constatar a plena existência, de trabalhos que lançassem mão exclusivamente de elementos de caráter projetual, de maneira a medir as eventuais diferenças entre esse método e os métodos tradicionais de pesquisa. Assim, de fato, o que foi possível observar, até o momento, foi a associação eficaz e produtiva entre métodos tradicionais, em especial de natureza historiográfica, e métodos potencialmente projetuais - ou ao menos, a presença de elementos da prática projetual consistentemente empregados como parte de pesquisas acadêmicas (Lima et al, 2011, p.7).
Mais recentemente os mestrados profissionais abriram a possibilidade de conciliar análise e avaliação de questões referentes ao ambiente construído com proposição projetual em nível de pós-graduação stricto sensu, a partir de um problema de pesquisa que o projeto visa resolver. Ou seja, a dissertação nos moldes de um mestrado acadêmico é acompanhada de uma proposta de intervenção espacial para aquele problema estudado. Procura-se, assim, inserir a pesquisa científica e a reflexão teórico-metodológica na prática profissional.

Em suma, podemos entender a pesquisa na área de projeto como um profícuo campo de geração de conhecimentos que tem rebatimento direto na qualidade do ambiente construído de nossas cidades e edifícios e que o reconhecimento deste campo como objeto de estudos científicos, inclusive pelas agências de fomento, tem permitido avanços significativos nos últimos 12 anos delineando um quadro mais favorável em relação à situação encontrada em 2003, quando da primeira edição do Seminário PROJETAR.

\section{A PESQUISA NA ÁREA dE PROJETO NA PRODUÇÃO DOS SEMINÁRIOS PROJETAR 2003-2013}

As seis primeiras edições dos Seminários PROJETAR produziram 857 artigos publicados em anais, conforme sintetizado na tabela 1 a seguir. Para análise das questões centrais tratadas neste artigo, fizemos inicialmente uma busca por palavras-chave nos anais de cada uma das edições, procurando primeiramente pelo termo "pesquisa" como indicador de que o artigo ou trata deste tema ou se apresenta como produto de uma pesquisa na área. Os resultados foram bastante decepcionantes quanto a este aspecto uma vez que apenas 21 (ou 2,4\%) dos 857 artigos indicaram pesquisa em suas palavras chaves. Para termos um contraponto apenas, ressalta-se que a palavra "ensino", sozinha ou acompanhada de ensino do projeto e correlatos, foi nominada em mais da metade do total de artigos (55\%). Essa foi uma primeira aproximação da questão, bastante limitada, mas mesmo assim indicadora do fato de se raramente mencionar "pesquisa" em palavras que deveriam indicar sinteticamente os assuntos ou conteúdos dos trabalhos. 
Tabela 1: Artigos publicados nos Anais das edições anteriores dos Seminários PROJETAR

\begin{tabular}{c|r|c|c|c} 
Edição/Ano & Total artigos & $\begin{array}{c}\text { Artigos com termo } \\
\text { "pesquisa" indicado nas } \\
\text { palavras-chave }\end{array}$ & $\begin{array}{c}\text { Artigos que se } \\
\text { apresentaram como } \\
\text { produtos de } \\
\text { pesquisas na área de } \\
\text { projeto (Resumos) }\end{array}$ & $\begin{array}{c}\text { Focos temáticos principais } \\
\text { dos artigos produtos de } \\
\text { pesquisa }\end{array}$ \\
\hline I PROJETAR/2003 & 91 & 02 & 42 & Ensino/Metodologia \\
\hline II PROJETAR/2005 & 146 & 06 & 87 & Ensino/Práticas \\
\hline III PROJETAR/2007 & 95 & 00 & 38 & Patrimônio/Ensino/Práticas \\
\hline IV PROJETAR/2009 & 243 & 06 & 176 & Ensino/Análise de obras \\
\hline V PROJETAR/2011 & 167 & 04 & 123 & Metodologia/Ensino \\
\hline VI PROJETAR/2013 & 115 & 03 & 59 & $\begin{array}{c}\text { Ensino/Metodologia/ } \\
\text { Análise de Obras }\end{array}$ \\
\hline Totais & 857 & 21 & 525 & \\
\hline
\end{tabular}

Uma segunda busca foi realizada por sessão, eixo temático ou mesa redonda que apresentasse o tema "Pesquisa" na programação dos eventos. Também foi relativamente baixa a incidência deste tipo de sessão, principalmente quando comparada às sessões sobre ensino, mas também às de práticas projetuais ou de extensão acadêmica. A partir daí, voltamos ao universo global de artigos, e com base na busca textual de palavras como "pesquisa" e "investigação" (no meio dos textos e não só em palavras chaves), identificamos aqueles que se apresentaram claramente como produtos de pesquisa na área de projeto (incluídas as desenvolvidas como dissertações de mestrado e teses de doutorado e aquelas relacionadas à análise de experiências práticas ou de ensino). Foram identificados 525 artigos que se disseram frutos de pesquisa, o que equivale a $61,2 \%$ do total produzido nestes seis encontros. Em seguida, selecionamos alguns artigos mais representativos para leitura, em primeiro lugar dos resumos e depois de seu conteúdo na íntegra, processo longo ainda em desenvolvimento.

A análise qualitativa desses materiais já permite, no entanto, a identificação de alguns aspectos de interesse para a construção de um panorama da pesquisa em projeto nos últimos 12 anos.

1) Quantos aos focos ou recortes temáticos dos artigos:

Em primeiro lugar, é notória a prevalência de pesquisas e discussões sobre ensino de projeto, seja com relatos e análises de experiências didáticas ou experimentos de novas no âmbito do atelier, ou mesmo fora dele, como no caso de experiências didáticas em workshops de projetos de arquitetura e urbanismo, por exemplo;
Em segundo lugar, destacam-se os estudos sobre a produção de obras e projetos (como produtos) de arquitetos renomados nacionalmente ou em determinada região a partir essencialmente da pesquisa historiográfica;

Um terceiro nível de investigação muito frequente diz respeito ao emprego de novos recursos de representação e linguagem (os informacionais notadamente) na prática projetual e no âmbito da formação profissional, em especial quanto ao seu rebatimento no ensino/aprendizagem do projeto;

Também deve-se destacar uma importante produção de pesquisas sobre habitação e em especial sobre habitação de interesse social no âmbito dos Seminários PROJETAR, principalmente depois da implementação do programa governamental Minha Casa, Minha Vida.

Foram pouco expressivas pesquisas sobre concepção e processos de projeto de profissionais ou discentes, embora haja uma maior incidência deste tipo de investigação nas últimas três edições do PROJETAR.

2) Quanto aos suportes teórico-metodológicos e técnicas utilizados nessas pesquisas, destacamos:

Pesquisa documental e historiográfica (textos e projetos/desenhos);

Estudos de casos representativos para análise direta (através de visita in loco, principalmente, e secundariamente com medições e outros levantamentos técnicos);

Levantamento de dados primários junto a atores envolvidos conforme o caso (entrevistas e aplicação de questionários a profissionais arquitetos, docentes e discentes de AU). 


\section{Tipos de análises empreendidas:}

i) Morfológica/tipológica (com auxílio de ferramentas como a gramática da forma e a sintaxe espacial e/ou apoiadas nas teorias e tendências dos movimentos arquitetônicos e urbanísticos modernos e contemporâneos);

ii) Funcional/Uso (com auxílio de recursos como a APO e construção de diagramas funcionais, por exemplo);

iii) Tecnológica (Materiais e Sistemas Construtivos) ou Tectônica (tendência mais recente);

iv) Sustentabilidade, Conforto Ambiental e Eficiência Energética (com auxílio de softwares de avaliação de desempenho);

v) Relação do projeto com a escala urbana/Inserção no lugar (também com auxílio de ferramentas computacionais).

É comum a associação de mais de um tipo de análise.

Mais recentemente tem aumentado o número de artigos sobre arquiteturas digitais e emprego de programas computacionais para a geração de formas complexas, o que vai ao encontro das tendências contemporâneas onde se constata a proliferação de fóruns específicos sobre este tema.

\section{CONSIDERAÇÕES FINAIS}

Essa foi uma primeira leitura do rico material intelectual produzido pela comunidade científica participante dos Seminários PROJETAR 20032013, considerado indicador importante da pesquisa neste campo, o que requer evidentemente, dada a sua magnitude, um aprofundamento em uma pesquisa específica e com maior horizonte de tempo. No entanto, ela permitiu evidenciar o avanço, nos últimos 12 anos, das investigações que tem o projeto de AU como objeto e seu reconhecimento oficial como campo legítimo da pesquisa acadêmica ainda a ser mais explorado. As análises dos dados levantados permitiram concluir que ainda persistem divergências conceituais e metodológicas importantes no que se entende como pesquisa neste campo específico, assim como a hegemonia das discussões sobre o ensino/aprendizado do projeto em nível de graduação (formação profissional) no âmbito dos Seminários PROJETAR. Deve-se destacar o incremento de abordagens relacionadas a inovações tecnológicas e à geração de formas complexas atreladas à concepção e desenvolvimento os projetos. Enfim, constatamos que a pesquisa em projeto se ampliou e avança positivamente, assim como o número de grupos de pesquisa e de programas de pós-graduação que tem esse foco, mas ainda são necessários aprofundamentos e refinamentos conceituais e metodológicos para sua maturação epistemológica, o que só é a nosso ver possível com a associação da pesquisa aplicada (cada vez mais priorizada) a investigações de cunho teórico e metodológico que possam não só realimentar mas também fundamentar a natureza essencialmente prática do fazer projetual.

\section{AGRADECIMENTOS}

Aos bolsistas de iniciação científica (CNPq) Gabriela Ricarte, João Felipe e Weksley Cavalcante, meus fiéis auxiliares no cotidiano de nossas pesquisas e na atualização do PROJEDATA.

\section{REFERÊNCIAS}

CHUPIN, J.P. Les prochaines 40 années : le doctorat en architecture à la charnière des enjeux disciplinaires e professionnels. Trames. n. 15. Architecture et Modernité. pp.121-144, 2004.

BOUTINET, J.P. Antropologia do Projeto. Porto Alegre: Artmed, 2000. Trad.

CAPES. Documento de Área 2013, Arquitetura, Urbanismo e Design. Disponível no http://capes.gov.br/images/stories/download/avaliacaotrienal/Docs de area/Arquitetura_Urbanismo_e_Design_doc area_e comiss\%C3\%A30_16out.pdf

LAWSON, B. Como arquitetos e designers pensam. São Paulo: Oficina de Textos, 2011.

LIMA, A.G. et al. Indicadores da Pesquisa Acadêmica em áreas de prática projetual. In. V PROJETAR 2011, Anais... Belo Horizonte. v.1, s/p., 2011. MALARD, M.L. Alguns problemas de projeto ou de ensino de arquitetura. Disponível em http://www.arq.ufmg.br/eva/art006.pdf. Acessado em maio/2015.

VELOSO, M. ; ELALI, G.A. Por uma formação mais qualificada do professor de projeto de arquitetura no Brasil. In. PROJETAR 2003. Anais... Natal, v.1, s/p., 2003.

VELOSO, M.; MARQUES, S. A pesquisa como elo entre teoria e prática do projeto: alguns caminhos possíveis. Arquitextos, São Paulo, ano 8, n. 088.8, Vitruvius, set. 2007 <http://www.vitruvius.com.br/revistas/read/arquitextos/08.088/211>.

\section{NOTAS}

(1) http://www.designresearchsociety.org/joomla/index.php; http://www.leap.umontreal.ca/; http://www.marseille.archi.fr/recherche/projects/ (2) http://www.fau.ufrj.br/prolugar/; http://www.mackenzie.br/511.html 